\title{
Ano-coccygeal support in the treatment of idiopathic chronic posterior anal fissure: a prospective non-randomised controlled pilot trial
}

\begin{abstract}
Background: Idiopathic chronic anal fissure is believed to be a consequence of a traumatic acute anodermal tear followed by recurrent inflammation and poor healing due to relative tissue ischaemia secondary to internal sphincter spasm. This pilot trial compared the efficacy of a novel manufactured ano-coccygeal support attached to a standard toilet seat (Colorec) to the standard procedure of lateral internal sphincterotomy (LIS) for chronic anal fissure. Methods: Fifty-three patients with confirmed chronic anal fissures were enrolled and assigned, based on their preference, to the test group and the control group. Each patient was reviewed after therapy, and follow-up was scheduled at 4, 6 and 8 weeks and at 6 months. Results: The fissure healing rate was $100 \%$ in both groups. There were no statistically significant differences between the test group $(n=30$, median age 42 years; range 201017 years) and the control group ( $n=22$, median age 38 years; range $23 \ddot{i} 60$ years) with regards to resolution of rectal bleeding at defaecation after 4 weeks ( 86.6 vs $72.7 \%, p=0.698)$, and by week 6 , bleeding had resolved in $100 \%$ of patients in both groups. There was no statistically significant difference between the test group and the control group with regards to pain scores at 4,6 and 8 weeks $(4.30 \pm 0.79,2.03 \pm 0.80,0.43 \pm 0.50$ vs $3.50 \pm 0.74,1.68 \pm 0.56,0.50 \pm$ $0.51, \mathrm{p}=0.054)$ and to time until complete healing of fissures $(5.60 \pm 1.52$ weeks vs $5.91 \pm$ 1.57 weeks, $\mathrm{p}=0.479$ ). After continuous use of the ano-coccygeal support over 6 months, no patients in the test group had recurrent fissures. No complications were observed during the trial. Conclusions: Results of both methods were comparable and demonstrated that the anococcygeal support is at least as effective as LIS, without any short-term complications. Larger and randomised trials on the use of ano-coccygeal support for chronic anal fissures are awaited.
\end{abstract}

Keyword: Ano-coccygeal support; Chronic posterior anal fissure; Lateral internal sphincterotomy; Medical therapy; Rectal bleeding 